\title{
General practitioner management of mental health during the COVID-19 pandemic
}

Ashvini Munindradasa, Grant Blashki, Sally Hall Dykgraaf, Jane Desborough, Michael Kidd

\section{Background}

Psychological issues are common presentations in general practice. The COVID-19 pandemic has created a 'perfect storm' for the deterioration of mental health. General practitioners (GPs) are ideally placed to provide supports for people with mental health issues.

\section{Objective}

The objective of this article is to provide an overview of management approaches for mental health issues, paying attention to fostering resilience and lifestyle factors, particularly during the COVID-19 pandemic period.

\section{Discussion}

While feelings of fear and anxiety are expected in these uncertain times, some people may experience an exacerbation of a mental illness or develop a mental health issue for the first time during the COVID-19 pandemic. A list of resources available to GPs is provided, and case studies are used to demonstrate how these resources can be used to support assessment and management during the COVID-19 pandemic.
MENTAL HEALTH ISSUES are common in the Australian community, with approximately 3.2 million Australians experiencing an anxiety-related condition and 2.5 million experiencing feelings of depression each year. ${ }^{1}$ The COVID-19 pandemic has brought fears relating to the virus itself (eg potential exposure of self and family), anxiety about physical distancing and concerns regarding unemployment and economic loss. ${ }^{2}$ Since the start of the pandemic, the Australian Government has announced that more than $\$ 500$ million will go towards boosting key mental health services, supporting the National Mental Health and Wellbeing Pandemic Response Plan. ${ }^{3}$ Unsurprisingly, general practitioners (GPs) have been called upon to provide increased mental health supports for their patients, further adding to their significant clinical workload.

The COVID-19 pandemic, lockdowns, associated loss of employment and financial stresses, navigation of homeschooling for parents while working from home and isolation of school-age children from their peer groups created a 'perfect storm' for the exacerbation of existing mental health conditions and the development of depression or anxiety. Further consequences included a reported spike in domestic violence and an increase in reported alcohol consumption. ${ }^{4,5}$ In some parts of Australia, COVID-19 closely followed devastating bushfires, meaning some communities were already in a heightened state of anxiety and stretched beyond their normal capacity. ${ }^{2,6}$

In high-income countries, GPs are central to providing mental healthcare, ${ }^{7}$ and are the first point of contact for mental health presentations and the facilitators of access to other therapists. ${ }^{89}$ Psychological issues are the most common health issues managed by Australian GPs. ${ }^{10}$ GPs often have longstanding relationships with their patients, and are well placed to understand their personal, family and community contexts and appreciate the interaction of concurrent physical and other mental health conditions and medication use.

With increased demand for mental health services during the pandemic, GPs need to provide increased mental health supports while keeping up to date with pandemic-specific guidelines and adapting to new methods of delivering healthcare. Furthermore, GPs themselves are experiencing similar worries and uncertainties related to potential infection of self and family, conflicting home and work commitments, as well as financial implications of the pandemic on practice viability. This article aims to offer guidance for GP management of depression and anxiety during the COVID-19 pandemic 
by providing an overview of management approaches (Figure 1), a list of resources (Table 1) and two case studies.

\section{Assessment \\ Apart from assessing presenting complaints and personal and family history of mental health conditions, a psychosocial history (ie coping methods, social supports and socio-occupational functioning) is essential to understand the full impact of the pandemic on an individual's mental health. ${ }^{11}$ Screening for mental health issues is particularly important for those with pre-existing mental health problems, healthcare workers, those in quarantine and the unemployed and casualised workforce, all of whom are known to be at increased risk of mental illness during a public health emergency. ${ }^{2}$ A mental health risk assessment should be considered in vulnerable patients for early detection of mental health problems or even suicidal thoughts. ${ }^{12}$ It should be noted that some people may present having already assessed their mental health by using tools available online. ${ }^{13-15}$ Regular follow-up to reassess symptoms and ensure that diagnoses and interventions are appropriate is important, as mental health issues can deteriorate over time.}

\section{Management \\ Fostering resilience}

The presence of increased anxiety, loneliness and sadness are expected in the context of a pandemic, and is not necessarily diagnostic of a mental health condition. ${ }^{2,16}$ GPs can help patients keep perspective and encourage positive behaviours, such as keeping up connections with friends and family, even in the presence of worry, pessimism and negative emotions about the pandemic.

While family resilience frameworks emphasise communication, organisation and belief systems, these are likely to be disrupted during a pandemic. ${ }^{17}$ GPs can help their patients reinforce such processes by focusing on the three Rs: routines, rituals and rules. ${ }^{17}$ Maintaining routines, developing new rituals and renegotiating rules so that they are still applicable during the pandemic can strengthen shared family values. ${ }^{17}$

Other models developed to manage emotional responses to natural disasters focus on the three Cs: control, coherence and connectedness. ${ }^{18}$ Control is reflected in the belief that people can access personal resources to achieve valued goals. Strengths-based cognitive behavioural therapy (CBT) can also be used to facilitate control, with patients encouraged to construct personal models of resilience that identify personal strengths, and then use metaphors and imagery to navigate challenges. ${ }^{19}$ Coherence is related to people's desire to make sense of the world. ${ }^{18}$ Encouraging a view that families are 'in it together', and that their current plight is specific, not the fault of anyone and time limited and manageable, may reinforce beneficial belief systems. ${ }^{17}$ Despite the impact of social distancing, isolation or quarantine requirements, connectedness can be emphasised with fewer people with whom patients have more meaningful relationships, or by expanding social

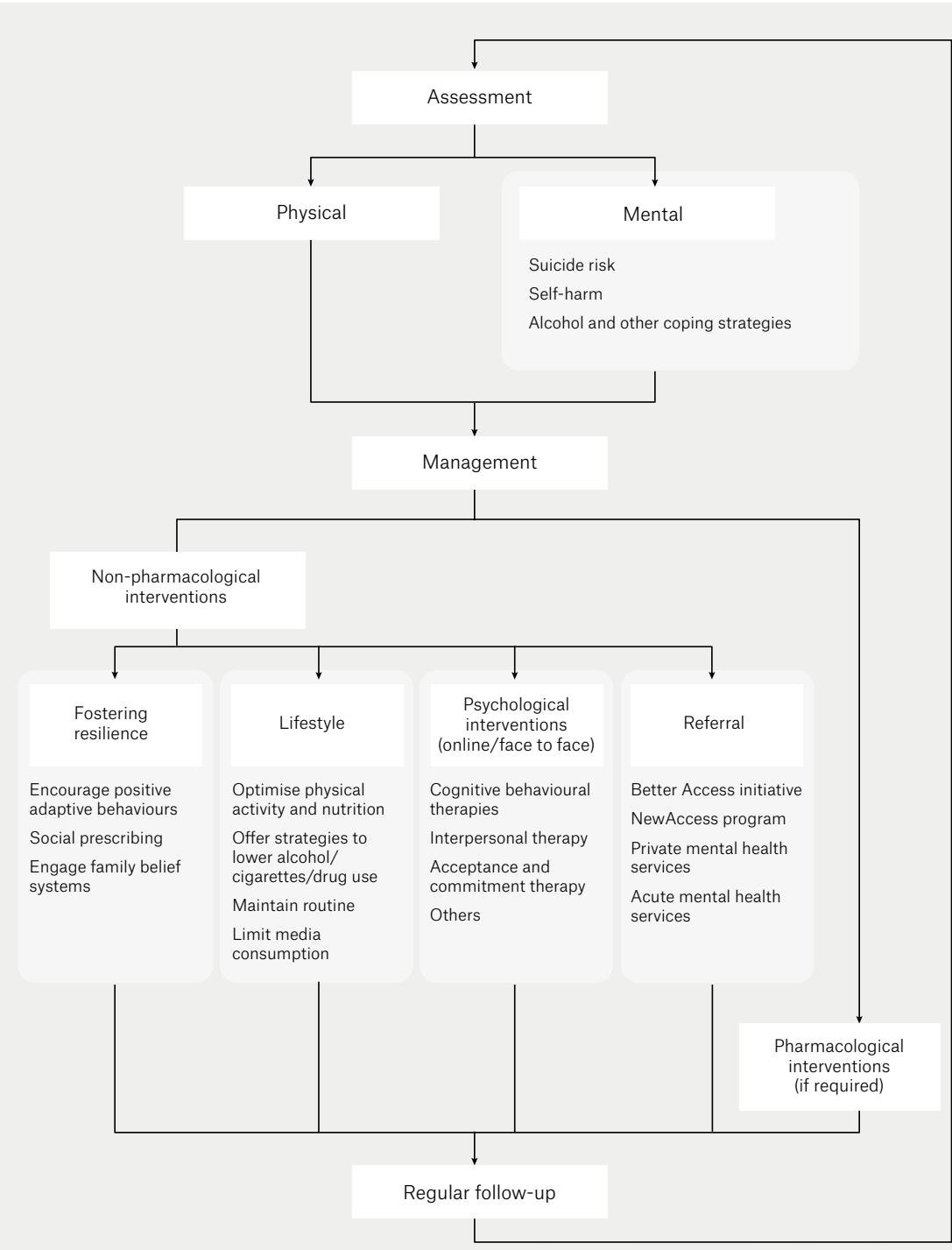

Figure 1. Overview of management approaches for mental illness during the COVID-19 pandemic 
contacts in ways that are not dependent on face-to-face engagement. ${ }^{18}$ Finally, 'social prescribing', where people are referred to non-clinical services, support groups and social structures, can be helpful for people experiencing mental health issues, chronic physical health conditions or social isolation. ${ }^{20,21}$

\section{Lifestyle}

Tobacco smoking, inadequate nutrition, harmful alcohol consumption and physical inactivity are behaviours that contribute to greater chronic disease burden, including for those with mental health issues. ${ }^{22}$ Optimising aspects of physical health, by focusing on healthy eating and regular exercise, should be discussed..$^{23,24}$ More minutes of daily exercise and more days per week spent outside in the sunshine for at least 10 minutes have been found to predict greater resilience during COVID-19. ${ }^{25}$ Coping mechanisms and strategies to minimise cigarette and alcohol use should be addressed. The Australian Government's Head to Health website provides helpful resources on physical activity, nutrition and sleep. ${ }^{26}$ Media reports can be a source of stress, and patients should be encouraged to monitor and limit their media consumption and rely on official and trusted sources of information. . $23,27^{2}$

\section{Psychological interventions} Several psychological interventions can assist people with mental health conditions. Favourable results have been reported for CBT, interpersonal psychotherapy (IPT) and acceptance and commitment therapy, even when delivered remotely and in multiple contexts, ${ }^{28-30}$ as is often necessary during the pandemic. CBT aims to modify maladaptive, automatic, negative thoughts and can reframe fears relating to hopelessness about social connection or fear of infection, whereas IPT allows the pandemic to be framed as a role transition. ${ }^{31}$ Promisingly, CBT, IPT and computer-assisted therapies have good evidence of effectiveness for common mental health conditions such as depression and anxiety. ${ }^{32,33}$

\section{Referral}

Most GPs are familiar with the Better Access to Psychiatrists, Psychologists and General Practitioners Initiative (Better Access), funded through the Australian Government's Medicare

\section{Table 1. Useful resources for Australian general practitioners}

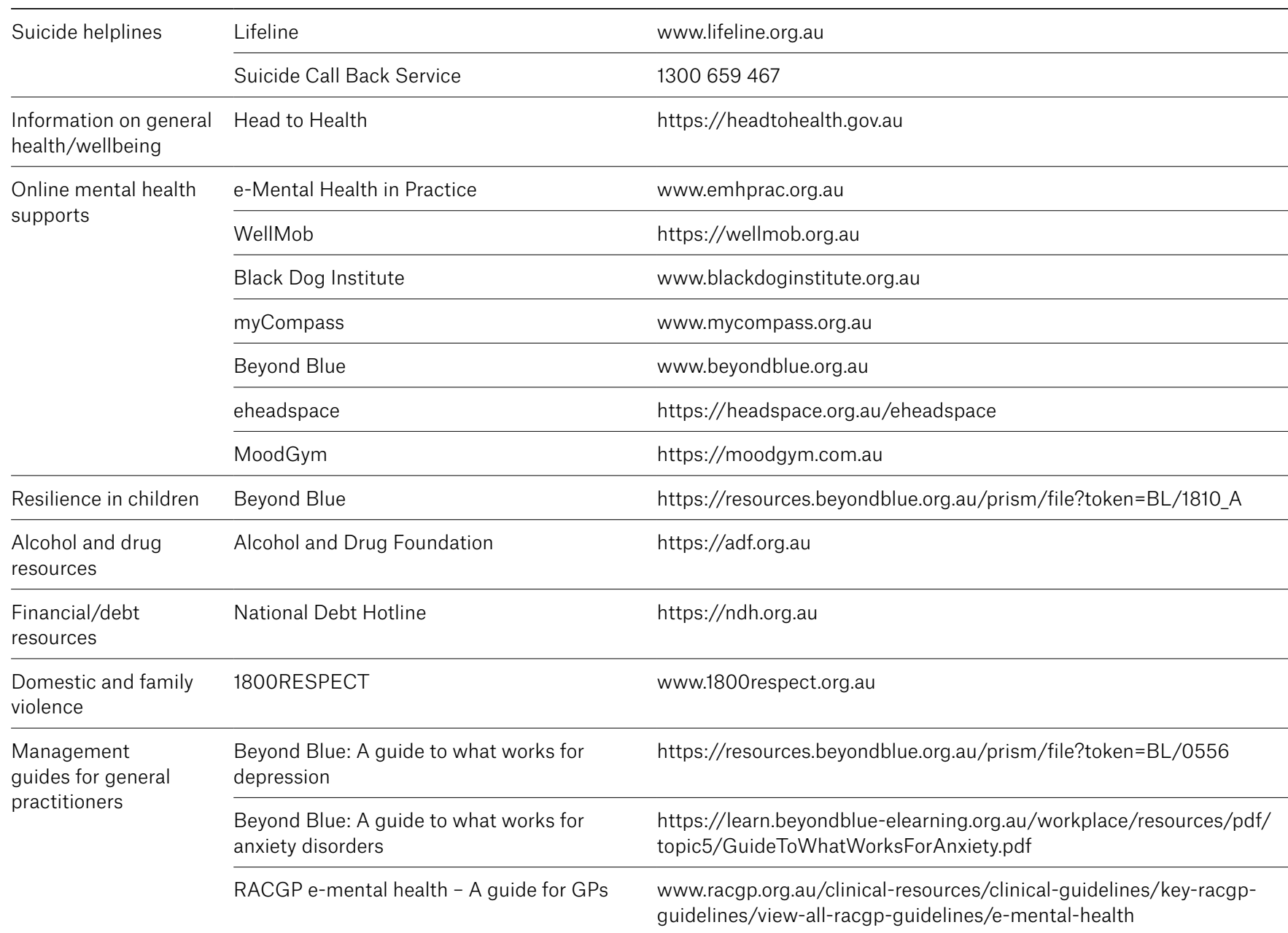


Benefits Schedule. During the pandemic, recognising the immense value of these psychology sessions, the Australian Government extended the program funding up to 20 sessions per calendar year. ${ }^{34}$ For people with milder symptoms, another option is referral to coaches in the NewAccess program, developed by Beyond Blue and available in a number of regions of Australia (Queensland, New South Wales, Victoria and the Australian Capital Territory at the time of writing).$^{35}$ People can self-refer to NewAccess for a total of six sessions and do not need a mental healthcare plan. ${ }^{35}$

\section{Pharmacological interventions}

While specific pharmacological therapies are outside the scope of this article, a few basic principles are worth reiterating. Knowing a few medications well (ie the short- and long-term side effect profiles, medication interactions and medicationdisease interactions) enables prescribers to be more confident in starting medications when needed. Once medications are started, patients should be followed up to monitor their progress and potential side effects. Special groups of people that should be treated with increased caution include older people, people with chronic disease, pregnant/breastfeeding women, children and adolescents. ${ }^{11} \mathrm{~A}$ detailed discussion of the various pharmacotherapies is outlined in the Psychotropic section of the Therapeutic Guidelines. ${ }^{36}$

\section{CASE 1}

Bob, aged 54 years, is a patient you know well. He presents three months after the date for his scheduled review of diabetes management. He has a history of metabolic syndrome and major depressive disorder. He lives alone. His main supports are his siblings, but they live in another state. Bob reports that he has been feeling down, and although he denies any active suicidal plans, admits that 'getting Corona might be a blessing'. He remains on the same dose of antidepressant medication and did not attend his last scheduled session with his psychologist, as it was over the telephone and 'what would be the point?' His investigations reveal glycated haemoglobin of $9.8 \%$.

Bob has a pre-existing mental health condition, is socially isolated and has a serious chronic physical health condition. Options for management include:

- assessment of symptoms using tools, such as Patient Health Questionnaire-9 (PHQ9), General Anxiety Disorder-7 (GAD7) or Depression, Anxiety and Stress Scales-21 (DASS-21)

- reiterating Bob's suicide safety plan

- emphasising the role of the 3Rs. Encourage Bob to stick to a regular routine (eg getting dressed at the same time every day and taking medication at the same time). Bob can develop new rituals, such as having dinner 'virtually' with his siblings a few times a week. New rules, such as having an hour of 'digital detox' or a 10-minute walk every day, can be trialled

- consideration of social prescribing of an activity that interests Bob. Examples of potential activities include walking, gardening, speaking to his siblings over the telephone, doing a shared project with his siblings (eg genealogy, cooking using television cooking shows/online recipes as a guide, painting or drawing that he can be encouraged to bring to appointments or taking courses/ volunteering with the local men's shed

- discussion about lifestyle interventions, such as physical activity and Bob's current diet, that are likely contributing to poor diabetes control. It may be worth discussing that improving Bob's diet and exercise may help improve his mental health as well

- making sure Bob's vaccinations are up to date, especially influenza and COVID-19 vaccinations

- encourage mindful engagement in activities and referring Bob to the Head to Health website for further information

- discussion about current coping mechanisms (eg alcohol consumption, smoking) and providing relevant supports

- reassuring Bob that psychological interventions conducted remotely are still effective, and encouraging Bob to re-engage with psychology
- consideration of whether Bob's dose of antidepressant requires adjustment

- follow-up options, including the use of telehealth.

\section{CASE 2}

Linda, aged 20 years, presents to you requesting sleeping tablets for insomnia, which she has experienced for the past few months. She was made redundant from her job at a local cinema where she was working to support herself as a musician, but all her shows were cancelled. She has a supportive family who are helping her apply for other jobs. She denies any suicidal ideation or thoughts of self-harm. Options for management include:

- discussion about the importance of sleep hygiene and routine in sleep. Linda can be referred to the Head to Health website to access the 'Managing insomnia course' or the 'Recharge app', which is a six-week wellbeing course that focuses on improving sleep habits

- trialling the $3 \mathrm{Cs}$ approach. Control can be fostered by encouraging Linda to focus on things she can do now to help with her short- and long-term goals. Linda can be directed to mindfulness apps, which may help her find coherence by acceptance-based practice of observing and engaging in the present in a non-judgemental and non-reactive manner. Linda can even start a virtual concert with fellow musicians to facilitate connectedness among her peers

- consideration of social prescribing (eg focusing on her music, walking groups with friends or volunteering to help with elderly residents in the community)

- discussion about the importance of healthy lifestyle interventions and encouraging mindful engagement in activities

- referral to the Beyond Blue website for more information and resources

- discussion and supports around any negative coping strategies

- referral to the NewAccess program for mental health coaching. 


\section{Conclusion}

Despite the various disruptions of the COVID-19 pandemic, most Australians are resilient and will not experience a diagnosable mental health condition. Feelings of fear and anxiety should not necessarily be considered pathological or require intervention; however, many others will have experienced an exacerbation of mental health concerns or developed a mental health condition for the first time. The management approach outlined in this article focuses on fostering resilience and promoting healthful lifestyle behaviours, psychological interventions and appropriate pharmacotherapy and referral. While this overview draws on online and applicationbased resources, the list of resources is not exhaustive, and clinicians have many options available to them to manage their patients' mental health in the context of the COVID-19 pandemic.

\section{Key points}

- GPs are well placed to manage mental health issues in the context of a pandemic.

- Anxiety, loneliness and sadness are normal responses to a pandemic and are not necessarily diagnostic of mental illness.

- Strategies, such as strengths-based CBT and social prescribing, can be used to foster resilience in people with mental illness who are experiencing social isolation.

- Lifestyle recommendations should include physical activity, healthy eating, reducing stress, strengthening social supports and promoting functioning in daily activities.

- Psychological interventions have been delivered remotely with favourable results.

\section{Authors}

Ashvini Munindradasa MChD, MPH, Academic General Practice Registrar, Rural Clinical School, Australian National University, ACT

Grant Blashki MBBS, MD, FRACGP, Lead Clinical Advisor, Beyond Blue, Vic; Associate Professor, Nossal Institute for Global Health and Melbourne Sustainable Society Institute, The University of Melbourne, Vic

Sally Hall Dykgraaf RN, Grad Cert Clin Man, PhD Scholar, Action Research, COVID-19 Primary Care
Response Group (seconded), Primary Care Division, Australian Government Department of Health, ACT; Research Manager, Rural Clinical School, ANU Medical School, College of Health and Medicine, Australian National University, ACT

Jane Desborough RN, RM, MPH, PhD, Action Research, COVID-19 Primary Care Response Group (seconded), Primary Care Division, Australian Government Department of Health, ACT; Senior Research Fellow, Research School of Population Health, Australian National University, ACT Michael Kidd AM, MD, FAHMS, Deputy Chief Medical Officer and Principal Medical Advisor, Primary Care Division, Australian Government Department of Health, ACT; Professor of Primary Care Reform, Australian National University, ACT; Adjunct Professor, Department of Family a Community Medicine, University of Toronto, ON; Emeritus Director, World Health Organization Collaborating Centre on Family Medicine and Primary Care, GVA; Professorial Fellow, Murdoch Children's Research Institute, The Royal Children's Hospital Melbourne, Vic; Honorary Professor of Global Primary Care, Southgate Institute for Health, Society and Equity, Flinders University, SA

Competing interests: None.

Funding: None.

Provenance and peer review: Not commissioned, externally peer reviewed.

Correspondence to:

ashvini.munindradasa@gmail.com

\section{Acknowledgements}

The authors would like to acknowledge the input of Tobias James and Alice Wetherell from the PHXchange, Research School of Population Health at the Australian National University in the development of the figures in this article.

\section{References}

1. Australian Bureau of Statistics. National health survey: First results. Belconnen, ACT: ABS, 2018. Available at www.abs.gov.au/statistics/health/ health-conditions-and-risks/national-healthsurvey-first-results/2017-18 [Accessed 14 April 2021].

2. Black Dog Institute. Mental health ramifications of COVID-19: The Australian context. Sydney, NSW: Black Dog Institute, 2020

3. Ministers Department of Health. Press release: Additional support for people with severe mental illness during the COVID-19 pandemic. Canberra, ACT: DoH, 2020. Available at www.health.gov. $\mathrm{au} /$ ministers/the-hon-greg-hunt-mp/media/ additional-support-for-people-with-severemental-illness-during-the-covid-19-pandemic [Accessed 14 April 2021].

4. Prime Minister of Australia. Media release: $\$ 1.1$ billion to support more mental health, Medicare and domestic violence services. Canberra, ACT: Prime Minister of Australia, 2020.

5. Biddle N, Edwards B, Gray M, Sollis K. Alcohol consumption during the COVID-19 period: May 2020. Canberra, ACT: The Australian National University, 2020.

6. Desborough J, Blashki G, Hall S, et al. Community activation, policy and COVID 19. In: World Federation for Mental Health. World Mental Health Day 2020. Mental health for all: Greater investment - greater access. San Antonio, TX: WFMH, 2020; p. 101-07.

7. Uwakwe R, Otakpor A. Public mental health Using the mental health gap action program to put all hands to the pumps. Front Public Health 2014;2:33. doi: 10.3389/fpubh.2014.00033.

8. Farrer LM, Walker J, Harrison C, Banfield M. Primary care access for mental illness in Australia: Patterns of access to general practice from 2006 to 2016. PLoS One 2018;13(6):e0198400-e. doi: 10.1371/journal.pone.0198400.

9. Australian Institute of Health and Welfare. Mental health services in Australia. Canberra, ACT: AlHW, 2021.

10. The Royal Australian College of General Practitioners. General practice: Health of the Nation 2020. East Melbourne, Vic: RACGP 2020.

11. World Health Organization. mhGAP intervention guide for mental, neurological and substance use disorders in non-specialized health settings. Geneva, CH: WHO, 2016

12. Su W, Stone L, Blashki G. Improving mental health and reducing suicide risk: How GPs can help during the COVID-19 pandemic. Med Today 2020;21(9):59-65.

13. Beyond Blue. Anxiety checklist. Melbourne, Vic: Beyond Blue, 2020. Available at www.beyondblue. org.au/the-facts/anxiety/anxiety-checklist [Accessed 14 April 2021].

14. Beyond Blue. Anxiety and depression checklist (K10). Melbourne, Vic: Beyond Blue, 2020. Available at www.beyondblue.org.au/the-facts/ anxiety-and-depression-checklist-k10 [Accessed 14 April 2021].

15. Black Dog Institute. Online clinic. Sydney, NSW: Black Dog Institute, 2019. Available at https:// onlineclinic.blackdoginstitute.org.au [Accessed 14 April 2021].

16. PeConga EK, Gauthier GM, Holloway A, et al. Resilience is spreading: Mental health within the COVID-19 pandemic. Psychol Trauma 2020;12(S1):S47-S8. doi: 10.1037/tra0000874.

17. Prime $\mathrm{H}$, Wade $\mathrm{M}$, Browne DT. Risk and resilience in family well-being during the COVID-19 pandemic. Am Psychol 2020;75(5):631-43. doi: 10.1037/amp0000660.

18. Polizzi C, Lynn SJ, Perry A. Stress and coping in the time of COVID-19: Pathways to resilience and recovery. Clin Neuropsychiatry 2020;17(2):59-62.

19. Padesky CA, Mooney KA. Strengths-based cognitive-behavioural therapy: A four-step model to build resilience. Clin Psychol Psychother 2012;19(4):283-90. doi: 10.1002/cpp.1795.

20. Howarth M, Griffiths A, da Silva A, Green R. Socia prescribing: A 'natural' community-based solution. Br J Community Nurs 2020;25(6):294-98. doi: 10.12968/bjen.2020.25.6.294.

21. The Royal Australian College of General Practitioners and Consumer Health Forum of Australia. Social prescribing roundtable, November 2019: Report. East Melbourne, Vic: RACGP and CHF, 2020.

22. Bartlem KM, Bowman JA, Bailey JM, et al. Chronic disease health risk behaviours amongst people with a mental illness. Aust N Z J Psychiatry 2015;49(8):731-41. doi: 10.1177/0004867415569798.

23. Sheridan Rains $L$, Johnson $S$, Barnett $P$, et al. Early impacts of the COVID-19 pandemic on mental health care and on people with mental health conditions: Framework synthesis of international experiences and responses. Soc Psychiatry Psychiatr Epidemiol 2021;56:13-24. doi: 10.1007/ s00127-020-01924-7.

24. Vinkers $\mathrm{CH}$, van Amelsvoort $\mathrm{T}$, Bisson Jl, et al. Stress resilience during the coronavirus pandemic. Eur Neuropsychopharmacol 2020;35:12-6. doi: 10.1016/j.euroneuro.2020.05.003. 
25. Killgore WDS, Taylor EC, Cloonan SA, Dailey NS. Psychological resilience during the COVID-19 lockdown. Psychiatry Res 2020;291:113216. doi: 10.1016/j.psychres.2020.113216.

26. Department of Health. Head to Health: COVID-19 Support. Canberra, ACT: DoH, 2020. Available at https://headtohealth.gov.au/covid-19-support/ covid-19 [Accessed 14 April 2021].

27. Pfefferbaum B, North CS. Mental health and the Covid-19 pandemic. N Engl J Med 2020;383(6):510-12. doi: 10.1056/NEJMp2008017.

28. Cuijpers P, Noma H, Karyotaki E, Cipriani A, Furukawa TA. Effectiveness and acceptability of cognitive behavior therapy delivery formats in adults with depression: A network meta-analysis. JAMA Psychiatry 2019;76(7):700-07. doi: 10.1001/ jamapsychiatry.2019.0268.

29. Dennis CL, Grigoriadis S, Zupancic J, Kiss A, Ravitz P. Telephone-based nurse-delivered interpersonal psychotherapy for postpartum depression: Nationwide randomised controlled trial. Br J Psychiatry 2020;216(4):189-96. doi: 10.1192/bjp.2019.275.
30. Kelson J, Rollin A, Ridout B, Campbell A. Internet-delivered acceptance and commitment therapy for anxiety treatment: Systematic review. J Med Internet Res 2019;21(1):e12530 doi: $10.2196 / 12530$

31. Swartz HA. The role of psychotherapy during the COVID-19 pandemic. Am J Psychother 2020;73(2):41-42. doi: 10.1176/appi. psychotherapy.20200015.

32. Morgan A, Reavley N, Jorm A, et al. A guide to what works for depression: An evidence-based review. 3rd edn. Melbourne, Vic: Beyond Blue, 2019.

33. Reavley N, Morgan A, Jorm A, et al. A guide to what works for anxiety disorders: An evidencebased review. 3rd edn. Melbourne, Vic: Beyond Blue, 2019.

34. Department of Health. Looking after your mental health during coronavirus (COVID-19) restrictions. Canberra, ACT: DoH, 2020. Available at www.health.gov.au/news/health-alerts/ novel-coronavirus-2019-ncov-health-alert/ ongoing-support-during-coronavirus-covid-19/ looking-after-your-mental-health-duringcoronavirus-covid-19-restrictions [Accessed 14 April 2021].

35. Beyond Blue. NewAccess - Mental health coaching support. Melbourne, Vic: Beyond Blue 2020. Available at www.beyondblue.org.au/getsupport/newaccess [Accessed 14 April 2021].

36. Expert Group for Psychotropic. Psychotropic. In: eTG complete [Internet]. West Melbourne, Vic: Therapeutic Guidelines Limited, 2021. 